\title{
Differential Expression of Adipocyte-Related Molecules in the Distal Epididymal Fat of Mouse during Postnatal Period
}

\author{
${ }^{\dagger}$ Ki-Ho Lee and Nan Hee Kim \\ Dept. of Biochemistry and Molecular Biology, College of Medicine, Eulji University, Daejeon 34824, Korea
}

\begin{abstract}
The epididymal fat of mouse is a part of visceral fat deposit and is divided into the distal or proximal part based on its histochemical characteristics. Even though the formation of the epididymal fat pad begins before the birth, a detailed adipogenic procedure of the epididymal fat has not been revealed. The epididymal fat pad becomes enlarged and expended with age, and expressional changes of numerous genes are associated with the maturation of fat tissues. In the present research, expressional patterns of adipose tissue-related genes in the distal epididymal fat of mouse at 2, 5, 8, and 12 months of postnatal age were determined by a quantitative real-time polymerase chain reaction (PCR) analysis. The lowest transcript levels of fatty acid binding protein 4 (Fabp4), lipoprotein lipase ( $L p l$ ), delta like non-canonical Notch ligand 1 (Dlkl), peroxisome proliferator-activated receptor gamma (Pparg), leptin (Lep), adiponectin (Adipoq), and resistin (Retn) were detected at 2 months of age, except fatty acid synthase (Fasn) showing the lowest level at 5 months of age. Even though expression of Lep and Fabp4 were gradually increased until 12 months of age, significant increases of Pparg and Adipoq transcript levels were continued until 8 months of age. The transcript levels of Lpl, Rent, Dlkl, and Fasn were significantly increased at 8 months of age, compared with those at 2 months of age. The current findings suggest that the expansion of the distal epididymal fat of mouse during postnatal period would be companied with differential expression of various adipocyte-associated molecules.
\end{abstract}

Key words : Distal epididymal fat, Gene expression, Adipose tissue, Postnatal development

\section{INTRODUCTION}

The epididymal fat belongs to visceral fat tissue, which is a part of gonadal adipose tissue and is placed between the testis and the head of epididymis (Pond, 1999; Niemala, 2008; Berry et al., 2013). The development of epididymal adipose tissue in the mouse appears to begin at the very early postnatal age and to be originated from non-adipose structure (Björntorp et al., 1979; Han et al., 2011). Lipidfilled adipocytes are detectable within the presumed epididymal fat at 7 days of postnatal age, and the maturation of adipocytes in the epididymal fat tissues continues afterward (Han et al., 2011). In addition to the lipid-filled adipocyte, the epididymal fat pad contains several nonlipid-filled cell types, including preadipocytes, vascular cells, and matrix cells (Cleary et al., 1977). The growth of epididymal fat during postnatal period accompanies with the increases of size and number of adipocyte (Cleary et al., 1977). Even though the increase of adipocyte number contributes to the increase of epididymal fat weight at the early postnatal ages, a change of epididymal fat weight

Manuscript received August 21, 2019, Received in revised form September 2, 2019, Accepted September 26, 2019

${ }^{\dagger}$ Corresponding Author : Ki-Ho Lee, Ph.D. Dept. of Biochemistry and Molecular Biology, Eulji University, Daejeon 34824, Korea. Tel: +82-42-259-1643, Fax: +82-42-259-1649, E-mail: kiholee@eulji.ac.kr

This is an Open Access article distributed under the terms of the Creative Commons Attribution Non-Commercial License (http:// creative-commons.org/licenses/by-nc/3.0) which permits unrestricted non-commercial use, distribution, and reproduction in any medium, provided the original work is properly cited. 
after 80 days of postnatal age is likely associated with the increase of adipocyte size (Cleary et al., 1977; Gruen et al., 1980).

The epididymal fat pad is histochemically divided into two regions, proximal and distal epididymal fat (Tirard et al., 2007). The proximal epididymal fat is located in direct contact with the testis and epididymis, while the distal epididymal fat is extended over the abdominal cavity (Tirard et al., 2007). Even though a clear morphological difference between proximal and distal epididymal fat has not been observed, there are some differences at histochemical and molecular biological levels (Tirard et al., 2007). For example, expression of aldo-keto reductase 1B7 in proximal epididymal fat is 10 times higher than that in distal epididymal fat (Tirard et al., 2007). In addition, expression of cyclooxygenase 2 and GATA-3 genes, specifically expressed in the pre-adipocyte, is higher in proximal epididymal fat than in distal epididymal fat (Tirard et al., 2007). These observations suggest that these two different epididymal fat parts could play different biological roles. Moreover, it is noteworthy that the removal of epididymal fat pad results in a cease of spermatogenesis (Chu et al., 2010). Thus, it is speculated that each epididymal fat part could involve in regulation of spermatogenesis in different manners

Several researches have examined differential gene expression of adipocyte-associated molecules in the epididymal fat pad at different postnatal ages (Liu et al., 2011; Lee \& Kim, 2018). Utilizing the adult mouse epididymal fat at 6, 14, and 18 months of postnatal age, Liu et al. (2011) have demonstrated that expression of fatty acid binding protein 4 (Fabp4), a gene involved in the differentiation of adipocyte, becomes reduced with age. In contrast, expression of peroxisome proliferator-activated receptor gamma (Pparg) in the mouse epididymal fat is increased with age (Liu et al., 2011). Interestingly, the expressional level of adiponectin (Adipoq) in the adult mouse epididymal fat is significantly decreased at 14 months of age but increased at 18 months of age, compared with that at 6 months of age (Liu et al., 2011). In the rat epididymal fat, expressional level of Pparg is significantly reduced with age (Lee \& Kim, 2018). However, unlike in the mouse epididymal fat, gene expression of Fabp4 becomes increased with age (Lee \& Kim, 2018). Together, these findings suggest that expressional patterns of molecules involved in differentiation to and maturation of adipocyte in the epididymal fat tissue would be dependent on species and postnatal ages.

Liu et al. (2011) have investigated gene expression of adipocyte-associated molecules in a whole epididymal fat at relatively old ages. However, in the present study, we had focused to examine expressional patterns of some adipocyte-related genes in the distal epididymal fat of mouse at different postnatal ages by quantitative real-time polymerase chain reaction (PCR) analysis.

\section{MATERIALS AND METHODS}

\section{Experimental design and isolation of the distal} epididymal fat pad

A total of 24 male C57BL/6N mice at 1 month of age were purchased from Samtako (OSan, Korea) and randomly divided into 4 experimental groups, including $2(\mathrm{n}=6), 5$ $(n=6), 8(n=6)$, and 12 months of age $(n=6)$. The mice were housed under controlled conditions and allowed for free access to food and water for experimental period. The present study was carried out in accordance with the guide for the care and use of laboratory animals of National Research Council in Korea.

Once reaching at a proper age, the animal was anesthetized by $\mathrm{CO}_{2}$ stunning, and the male reproductive tract, including the testis and epididymis, was collected through an incision on lower abdominal region. The isolated reproductive tract was immersed in cold-PBS solution, and the distal epididymal fat was carefully dissected out from the rest of the epididymal fat and other reproductive tissues. The distal epididymal fat was rinsed in fresh cold- 
PBS solution and quickly placed in an eppendorf tube to freeze in liquid nitrogen. Then, the distal epididymal fat was stored in $-80^{\circ} \mathrm{C}$ before the isolation of total RNA.

2. Isolation of total RNA and construction of complementary DNA (cDNA)

The distal epididymal fat isolated from a mouse was homogenized in $1 \mathrm{~mL}$ of TRI REAGENT solution (Molecular Research Center, Inc, Cincinnati, OH, USA). Total RNA pellet was extracted by sequential addition of chloroform and isopropanol. Air-dried total RNA pellet was resuspended in DEPC- $\mathrm{dH}_{2} \mathrm{O}$, and the concentration of total RNA was determined by with a NanoDrop Lite spectrophotometry (Thermo Scientific, Wilmington, DE, USA). The quality of total RNA extracted was examined by $1.2 \%$ agarose gel electrophoresis.

To perform reverse transcription (RT) reaction in iScript ${ }^{\mathrm{TM}}$ RT Supermix for RT-qPCR (Bio-Rad Laboratories. Inc., Hercules, CA, USA), $1 \mu \mathrm{g}$ of total RNA was mixed with oligo-dT primer, reverse transcriptase, $5 \mathrm{X}$ reaction buffer, and nuclease-free $\mathrm{dH}_{2} \mathrm{O}$ to make a final volume of $20 \mu \mathrm{L}$ of RT reaction solution. The RT reaction was carried out in a sequential order of $25^{\circ} \mathrm{C}$ for $5 \mathrm{~min}, 46^{\circ} \mathrm{C}$ for $20 \mathrm{~min}$, and $95^{\circ} \mathrm{C}$ for $1 \mathrm{~min}$. Then, the generated cDNA was used to determine expressional levels of target molecules in the mouse distal epididymal fat by quantitative real-time PCR analysis.

\section{Quantitative real-time polymerase chain reaction} analysis

The oligonucleotide primers for target genes examined by quantitative real-time PCR were designed with Primer 3 software (http://www.bioneer.co.kr/cgi-bin/primer/primer3. cgi: Whitehead Institute/MIT Center for Genomes Research, USA), and primers information is provided in Table 1.

Quantitative real-time PCR analysis was performed with a mixture of $1 \mu \mathrm{L}$ of cDNA, 10 pmol of each primer, $7 \mu \mathrm{L}$ of $\mathrm{iQ}^{\mathrm{TM}} \mathrm{SYBR}^{\circledR}$ Green Supermix (Bio-Rad Laboratories), and $\mathrm{dH}_{2} \mathrm{O}$ to make a final volume of $20 \mu \mathrm{L}$. The PCR was carried out in a condition of a pre-denaturation at $95^{\circ} \mathrm{C}$ for $5 \mathrm{~min}$, cycles of a denaturation at $95^{\circ} \mathrm{C}$ for $30 \mathrm{sec}$, an annealing at $\mathrm{T}_{\mathrm{m}}$ for $30 \mathrm{sec}$, and an extension at $72^{\circ} \mathrm{C}$ for 30 sec, and an additional extension at $72^{\circ} \mathrm{C}$ for $10 \mathrm{~min}$. As a PCR quality control purpose, $18 \mathrm{~S}$ ribosomal RNA (Rn18s) was included as an internal control. The size of PCR product was check with $1.2 \%$ agarose gel electrophoresis.

\section{Statistical analysis and presentation of data}

The transcript level of a target gene at a given postnatal age was normalized with that of Rn18s to get a mean and standard error derived from quadrupled or quintupled RT reactions and PCRs. The data were presented in a relative ration of expressional level of a target gene against Rn18s. One-way ANOVA analysis, followed by Duncan's test as a post-hoc analysis, was performed to determine the existence of statistical significance among expressional levels of a target gene at different postnatal ages. If $p>0.05$, it was considered that the expressional levels of a target gene at different postnatal ages were statistically significant.

\section{RESULTS}

\section{Gene expression of Lep and Pparg in the distal} epididymal fat of mouse during postnatal period

Expressional levels of leptin (Lep) in the mouse distal epididymal fat at different postnatal ages are shown in Fig. 1a. The lowest transcript level of Lep was observed at 2 months of age, followed by a significant increase of Lep transcript level at 5 months of age (Fig. 1a). The expressional level of Lep in the distal epididymal fat of mouse at 8 months of age was significantly higher than that of 5 months of age (Fig. 1a). An additional significant rise of Lep transcript level was detected at 12 months of age (Fig. 1a).

The expression of Pparg in the mouse distal epididymal fat at 5 months of age was significantly increased, compared with that at 2 months of age (Fig. 1b). A further increase of 
Table 1. Primers used for real-time PCR analysis

\begin{tabular}{|c|c|c|c|}
\hline Gene & $\begin{array}{l}\text { Primer sequence } \\
\qquad\left(5^{\prime} \rightarrow 3^{\prime}\right)\end{array}$ & $\begin{array}{l}\mathrm{T}_{\mathrm{m}} \\
\left({ }^{\circ} \mathrm{C}\right)\end{array}$ & $\begin{array}{l}\text { PCR product } \\
\text { size (bps) }\end{array}$ \\
\hline $\begin{array}{c}\text { Fabp4 } \\
\left(\mathrm{NM} \_024406.3\right)\end{array}$ & $\begin{array}{l}\text { (F) CAAGCCCAACATGATCATCAGC } \\
\text { (R) CACGCCCAGTTTGAAGGAAATC }\end{array}$ & 60 & 97 \\
\hline $\begin{array}{c}\text { Fasn } \\
(\text { NM_011480.3) }\end{array}$ & $\begin{array}{l}\text { (F) GTGTGGACATGGTCACAGATG } \\
\text { (R) GACCGCTTGGGTAATCCATA }\end{array}$ & 60 & 67 \\
\hline $\begin{array}{c}L p l \\
\left(\mathrm{NM} \_008509.2\right)\end{array}$ & $\begin{array}{l}\text { (F) TCAGAGCCAAGAGAAGCAGCA } \\
\text { (R) TTGTGTTGCTTGCCATCCTCA }\end{array}$ & 60 & 118 \\
\hline $\begin{array}{c}\text { Dlk1 } \\
\left(\mathrm{NM} \_010052.5\right)\end{array}$ & $\begin{array}{l}\text { (F) GGCCATCGTCTTTCTCAACA } \\
\text { (R) CTCCTCATCACCAGCCTCCT }\end{array}$ & 60 & 181 \\
\hline $\begin{array}{c}\text { Pparg } \\
(\mathrm{U} 01841.1)\end{array}$ & $\begin{array}{l}\text { (F) GATTCATGACCAGGGAGTTC } \\
\text { (R) GACTCTGGGTGATTCAGCTT }\end{array}$ & 60 & 247 \\
\hline $\begin{array}{c}\text { Lep } \\
\left(\mathrm{NM} \_008493.3\right)\end{array}$ & $\begin{array}{l}\text { (F) TTCACACACGCAGTCGGTAT } \\
\text { (R) TCATTGGCTATCTGCAGCAC }\end{array}$ & 55 & 165 \\
\hline $\begin{array}{c}\text { Adipoq } \\
\text { (NM_009605.5) }\end{array}$ & $\begin{array}{l}\text { (F) AGGCCGTTCTCTTCACCTACG } \\
\text { (R) GACTTGGTCTCCCACCTCCAGA }\end{array}$ & 62 & 95 \\
\hline $\begin{array}{c}\text { Retn } \\
\left(\mathrm{NM} \_022984.3\right)\end{array}$ & $\begin{array}{l}\text { (F) TTCCTTGTCCCTGAACTGCT } \\
\text { (R) TCAAGACTGCTGTGCCTTCT }\end{array}$ & 55 & 187 \\
\hline $\begin{array}{c}\text { Rn18s } \\
(\mathrm{NR} 03278.3)\end{array}$ & $\begin{array}{l}\text { (F) ACTCAACACGGGAAACCTCA } \\
\text { (R) AACCAGACAAATCGCTCCAC }\end{array}$ & 55 & 123 \\
\hline
\end{tabular}

PCR, polymerase chain reaction, Fabp4, fatty acid binding protein 4; Fasn, fatty acid synthase; $L p l$, lipoprotein lipase; Dlkl, delta like non-canonical Notch ligand 1; Pparg, peroxisome proliferator-activated receptor, gamma; Lep, leptin; Adipoq, adiponectin; Retn, resistin; Rn18s, 18S ribosomal RNA.

Pparg transcript level was detected at 8 months of age, without an additional significant change of Pparg transcript level at 12 months of age (Fig. 1b).

2. Gene expression of $L p /$ and Fabp4 in the distal epididymal fat of mouse during postnatal period

The expressional level of lipoprotein lipase $1(\mathrm{Lpl})$ in the mouse distal epididymal fat at 5 months of age was not significantly changed, compared with that at 2 months of age (Fig 2a). However, over 1-folding increase of $\mathrm{Lpl}$ transcript level in the distal epididymal fat of mouse was detected at 8 months of age (Fig. 2a). There was no significant change of $\mathrm{Lpl}$ expressional level at 12 months of age (Fig. 2a).

The expressional pattern of Fabp4 in the mouse distal epididymal fat during postnatal period is shown in Fig. $2 b$. The level of Fabp4 transcript at 5 months of age was significantly increased, compared with that at 2 months of age (Fig. 2b). An additional increase of Fabp4 transcript level was detected at 8 months of age, and the highest expressional level of Fabp 4 in the mouse distal epididymal fat was observed at 12 months of age (Fig. 2b). 
(a)

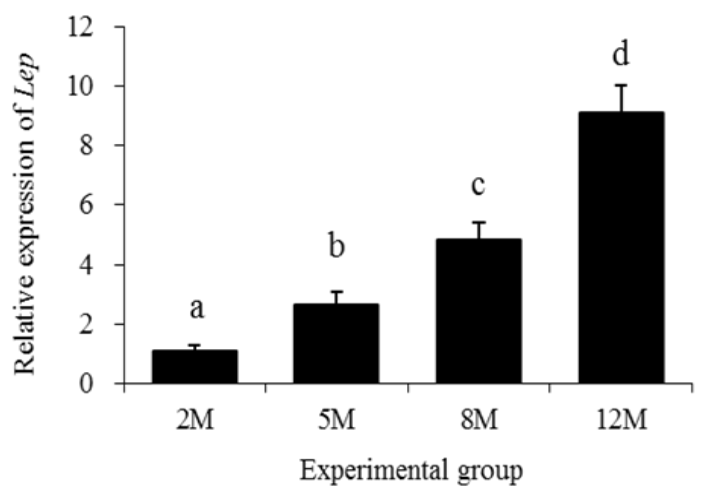

(b)

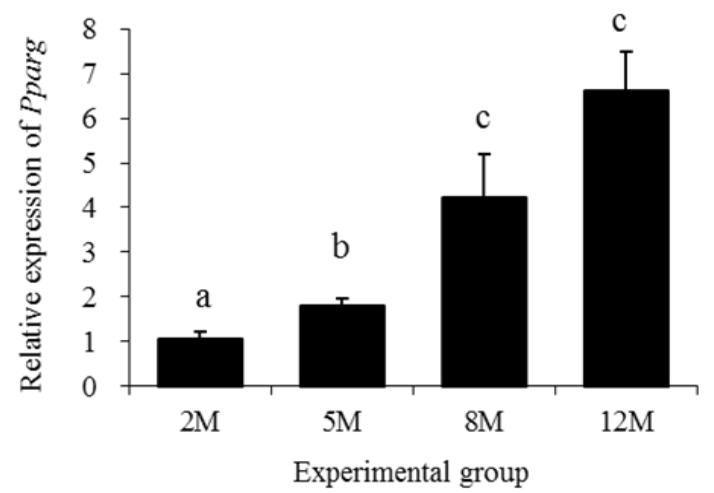

Fig. 1. Gene expression of Lep and Pparg in the distal epididymal fat of mouse during postnatal period. The relative transcript levels of Lep (a) and Pparg (b) are shown. Different letters indicate statistical significances at $p<0.05$. Lep, leptin; Pparg, peroxisome proliferator-activated receptor gamma; M, month.

(a)

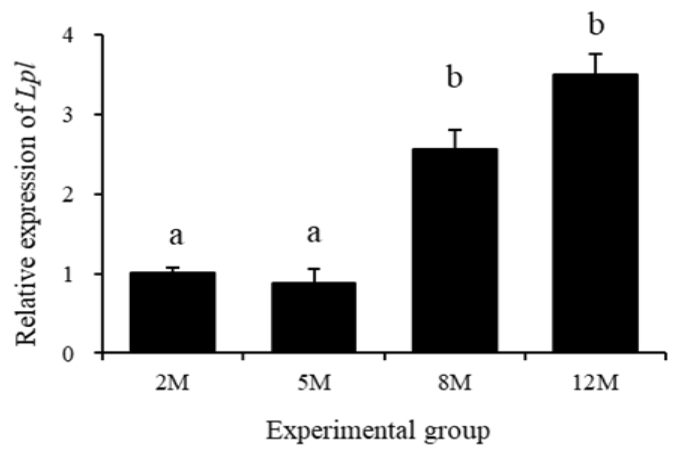

(b)

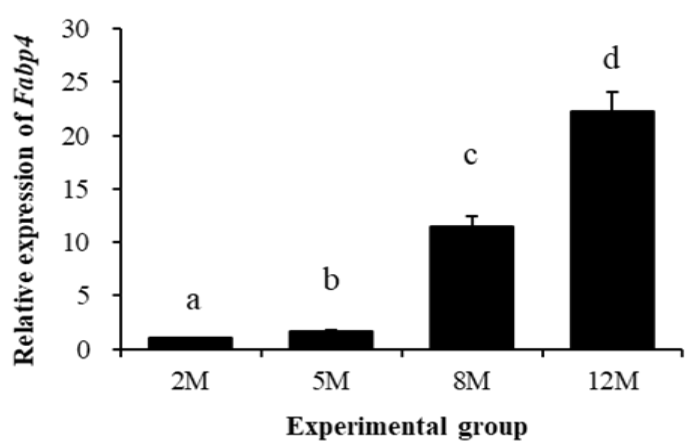

Fig. 2. Gene expression of $\mathbf{L P l}$ and Fabp4 in the distal epididymal fat of mouse during postnatal period. The relative transcript levels of $\mathrm{Lpl}$ (a) and Fabp4 (b) are shown. Different letters indicate statistical significances at $p<0.05$. Lpl, lipoprotein lipase; Fabp4, fatty acid binding protein 4; M, month.

\section{Gene expression of DIk1 and Rent in the distal} epididymal fat of mouse during postnatal period

The lowest expressional level of delta like non-canonical Notch ligand $1(D l k l)$ in the mouse distal epididymal fat was detected at 2 months of age (Fig. 3a). There was no significant change of $D l k l$ transcript level at 5 months of age, compared with that at 2 months of age (Fig. 3a). However, a tremendous increase of $D l k l$ transcript level in the mouse distal epididymal fat was found at 8 months of age, followed by an additional surge of $D l k l$ expression at 12 months of age (Fig. 3a).
The transcript level of resistin (Retn) in the mouse distal epididymal fat at 5 months of age was not significantly different with that at 2 months of age (Fig. 3b). But, the expressional level of Retn was significantly increased at 8 months of age (Fig. 3b). There was no significant difference of Retn transcript level in the mouse distal epididymal fat between at 8 months of age and at 12 months of age (Fig. 3b).

4. Gene expression of Adipoq and Fasn in the distal epididymal fat of mouse during postnatal period

Fig. 4 shows expressional patterns of Adipoq and fatty 
(a)

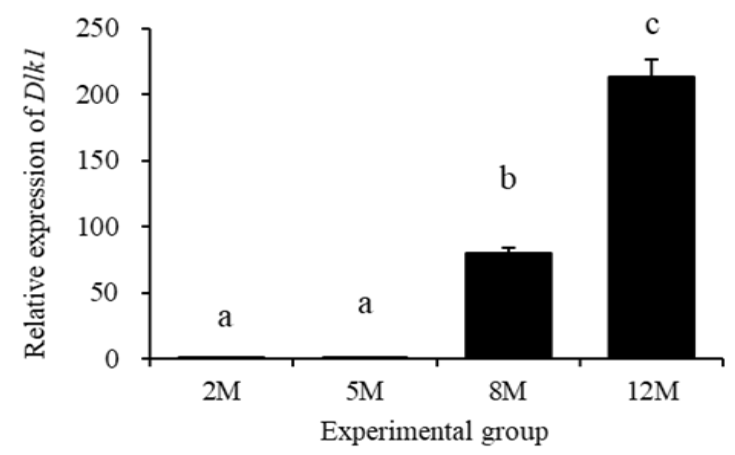

(b)

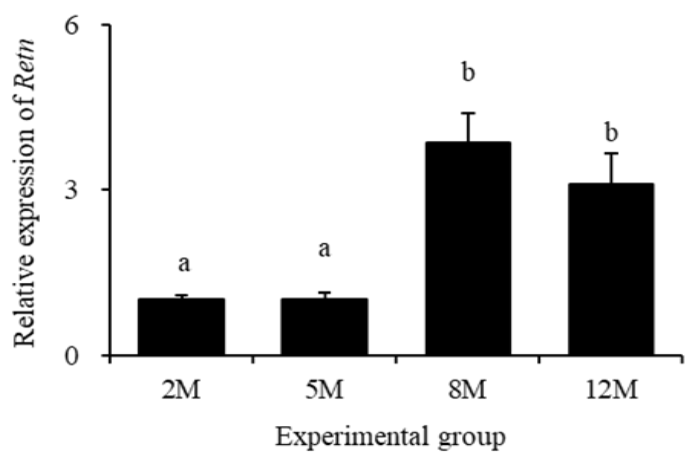

Fig. 3. Gene expression of Dlk1 and Rent in the distal epididymal fat of mouse during postnatal period. The relative transcript levels of Dlk1 (a) and Rent (b) are shown. Different letters indicate statistical significances at $p<0.05$. Dlkl, delta like non-canonical Notch ligand 1; Retn, resistin; M, month.

(a)

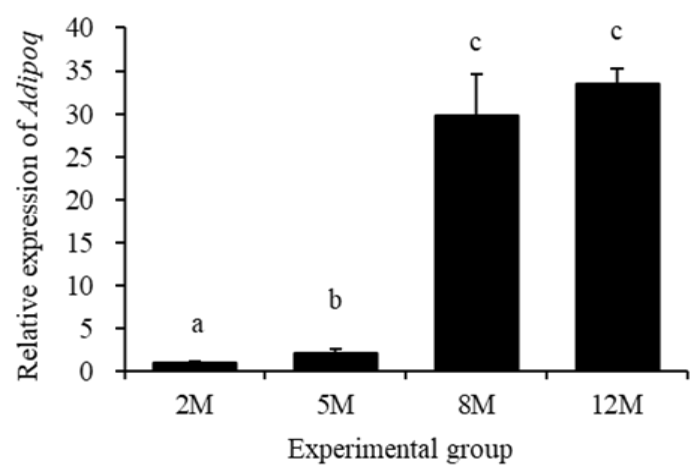

(b)

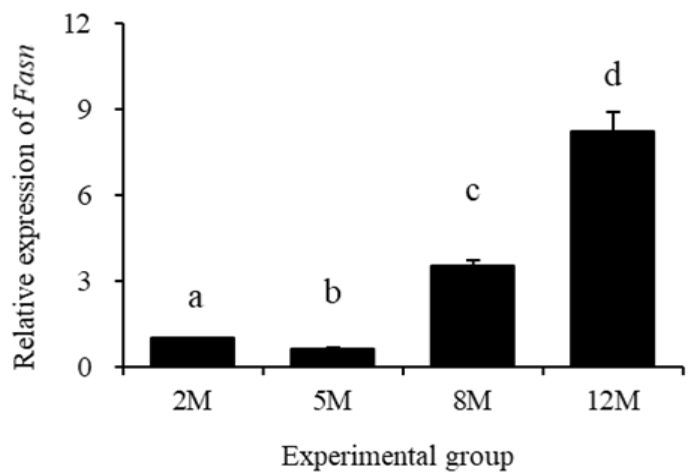

Fig. 4. Gene expression of Adipoq and Fasn in the distal epididymal fat of mouse during postnatal period. The relative transcript levels of Adipoq (a) and Fasn (b) are shown. Different letters indicate statistical significances at $p<0.05$. Adipoq, adiponectin; Fasn, fatty acid synthase; M, month.

acid synthase (Fasn) in the mouse distal epididymal fat at different postnatal ages. A significant increase of Adipoq transcript level was found at 5 months of age, compared with that at 2 months of age (Fig. 4a). Even though the expressional level of Adipoq at 8 months of age was significantly higher than that at 5 months of age, there was no significant change of Adipoq transcript level between at 8 months of age and 12 months of age (Fig. 4a).

The expressional level of Fasn in the mouse distal epididymal fat at 5 months of age was significantly decreased, compared with that at 2 months of age (Fig. 4b). However, the level of Fasn transcript at 8 months of age was significantly higher than that at 2 months of age (Fig. 4b). A further significant increase of Fasn transcript level was observed at 12 months of age (Fig. 4b).

\section{DISCUSSION}

The expression of molecules related with adipogenic differentiation and adipocyte secretion in the distal epididymal fat, not a whole epididymal fat pad, at different postnatal ages. Even though expressional patterns of all molecules examined are not exactly same, expressional levels of target molecules are generally low at 2 months of 
age and become increased with age.

The adipose tissue not only stores excess energy in a form of fatty acid but also secrets various cytokines, called adipocytokines, including Lep, Adipoq, and Reth (Cao, 2014; Haiming, 2014). Most of these adipocytokines involve in the regulation of energy metabolism at systemic level by providing a communication between adipose tissue and other metabolic tissues, such as liver, brain, and heart (Cao, 2014; Haiming, 2014). For example, Lep controls the secretion of several signal molecules from hypothalamus to regulate food intake and energy expenditure in the body (Cao, 2014). It is generally thought that Adipoq and Retn play opposite roles on insulinresistance and atherogenesis (Cao, 2014). Using a whole epididymal fat tissue of mouse, expression of Adipoq at 14 months of age is lower than that at 6 months of age (Liu et al., 2011). However, the present study has shown a significant increase of Adipoq expression at 12 months of age, compared with that at 5 months of age. Such opposite observations on expression of Adipoq from Liu et al. (2011) and present research could be due to the difference of tissue examined. As reported from Tirard et al. (2007) research, the proximal epididymal fat has distinguishable molecular characteristics from distal epididymal fat. Thus, it is reasonable to consider that expressional level of Adipoq within a whole epididymal fat could differ with that in the distal epididymal fat. The examination of expression of Adipoq in the proximal epididymal fat during postnatal period could be helpful to understand the regional differences of the epididymal fat. Our previous research has demonstrated steady increases of Lep and Retn transcript levels in the rat whole epididymal fat with age (Lee \& Kim, 2018), mostly in agreement with the present findings. Thus, it seems that increases of Lep and Retn expression in the epididymal fat occur generally with age.

The differentiation of pre-adipocyte into mature adipocyte and maintenance of differentiated state of adipocyte require expression of Pparg (Tamori et al., 2002). Our earlier research has shown that expression of Pparg in the rat epididymal fat at 12 months of age is lower than that at 5 months of age (Lee \& Kim, 2018). However, the level of Pparg transcript in a whole epididymal fat of mouse at 6 months of age becomes significantly increased at 14 months of age (Liu et al., 2011), similarly with our current findings. Hence, it is believed that expression of Pparg in the epididymal fat could appear in different manner among species. The examination of expressional patterns of Pparg in the mouse proximal epididymal fat during postnatal period would be helpful to understand expressional regulation of Pparg in a whole epididymal fat tissue of mouse.

A function of FABP4 is the promotion of lipid storage in adipogenic cells, and expression of Fabp4 in the epididymal fat is stimulated by an insulin-sensitizing element (Kletzien et al., 1992). Our previous study has a gradual increase of Fabp4 transcript level in the rat epididymal fat with age (Lee \& Kim, 2018). However, Liu et al. (2011) have reported a significant reduction of Fabp 4 transcript level in the mouse epididymal fat at 14 months of age, compared with that at 6 months of age. Interestingly, the current findings for expressional patterns of Fabp4 in the mouse distal epididymal fat during postnatal period are in strong agreement with our previous observations (Lee \& Kim, 2018). As described above in Adipoq section, the disagreement between current and Liu et al. (2011)' findings on Fabp4 expression could be due to the usage of different experimental tissue, a whole or distal epididymal fat. An additional research with the proximal epididymal fat of mouse is suggested to find an explanation for such conflicted observations.

The LPL is an enzyme catalyzing the hydrolysis of triglycerides, promoting lipolysis. It has been reported that the expression of $\mathrm{Lpl}$ in the rat epididymal fat tissue during postnatal period is decreased with age (Kirkland et al., 1993). However, the current research reveals that expression of $L p l$ in the distal epididymal fat of mouse is significantly 
increased at 8 months of age, even though there is no major change of $\mathrm{Lpl}$ transcript level at 12 months of age. Gruen et al. (1980) have shown that glycerol release per rat epididymal adipocyte is inversely related with fat cell size at the early postnatal age but correlates with fat cell size after 73 days of age, implying increased activity and expression of LPL in the rat epididymal fat pad with age. Gruen et al. (1980)'s findings seems to agree with data obtained from the present research. But, in spite of an increase of epididymal fat size, there is no significant change on expressional level of $\mathrm{Lpl}$ at 5 months of age, compared that at 2 months of age. As described earlier, because the proximal and distal epididymal fat of mouse has different molecular features, it is possible that the mouse proximal epididymal fat exhibits much higher expressional level of $\mathrm{Lpl}$ at 5 months of age. In opposite to LPL, FASN is a key enzyme involving in lipogenesis in adipose tissue. The FASN catalyzes palmitate synthesis in the adipocyte. Thus, it is presumable that increases of adipocyte in the size and number with age could result in an increase of Fasn expression. Indeed, expressional level of Fasn in the distal epididymal fat of mouse is increased with age, except a transient decrease of Fasn transcript level at 5 months of age. Such temporary change of Fasn expression could not be explained at this moment. If additional data derived from a study with the proximal epididymal fat are available, it would be possible to address overall expressional change of Fasn in the mouse epididymal fat during postnatal period.

In summary, the present research shows the expressional increase of selected adipocyte-associated molecules in the distal epididymal fat with age. Because of broad biological effects of molecules originated from the adipocyte, it is supposed that the altered expressional levels of adipocytesecreted elements in the distal epididymal fat with age could be linked with the onset of metabolic syndrome and/or the decline of reproductive capacity, which is a common condition frequently found at the late age.

\section{CONFLICT OF INTERESTS}

The authors declare no potential conflict of interest.

\section{ACKNOWLEDGEMENTS}

This research was supported by Basic Science Research Program through the National Research Foundation of Korea (NRF) funded by the Ministry of Education (2017R1D1A 1B03030209).

\section{REFERENCES}

Berry DC, Stenesen D, Zeve D, Graff JM (2013) The developmental origins of adipose tissue. Development 140:3939-3949.

Björntorp P, Karlsson M, Gustafsson L, Smith U, Sjöström L, Cigolini M, Storck G, Pettersson P (1979) Quantitation of different cells in the epididymal fat pad of the rat. J Lipid Res 20:97-106.

Cao H (2014) Adipocytokines in obesity and metabolic disease. J Endocrinol 220:T47-T59.

Cleary MP, Greenwood MR, Brasel JA (1977) A multifactor analysis of growth in the rat epididymal fat pad. J Nutr 107:1969-1974.

Gruen R, Kava R, Greenwood MR (1980) Development of basal lipolysis and fat cell size in the epididymal fat pad of normal rats. Metabolism 29:246-253.

Cao H (2014) Adipocytokines in obesity and metabolic disease. J Endocrinol 220:T47-T59.

Han J, Lee JE, Jin J, Lim JS, Oh N, Kim K, Chang SI, Shibuya M, Kim H, Koh GY (2011) The spatiotemporal development of adipose tissue. Development 138 5027-5037.

Kirkland JL, Hollenberg CH, Gillon W (1993) Ageing, differentiation, and gene expression in rat epididymal preadipocytes. Biochem Cell Biol 71:556-561. 
Kletzien RF, Foellmi LA, Harris PK, Wyse BM, Clarke SD (1992) Adipocyte fatty acid-binding protein: Regulation of gene expression in vivo and in vitro by an insulin-sensitizing agent. Mol Pharmacol 42:558-562.

Lee KH, Kim NH (2018) Expressional patterns of adipocyte-associated molecules in the rat epididymal fat during postnatal development period. Dev Reprod 22:351360.

Liu LF, Shen WJ, Ueno M, Patel S, Kraemer FB (2011) Characterization of age-related gene expression profiling in bone marrow and epididymal adipocytes. BMC Genomics 12:212.

Niemela S, Miettinen S, Sarkanen JR, Ashammakhi N (2008) Adipose tissue and adipocyte differentiation:
Molecular and cellular aspects and tissue engineering applications. In: Ashammakhi N, Reis R, Chiellini F (ed), Topics in Tissue Engineering, Vol. 4, pp 1-26.

Pond CM (1999) Physiological specialisation of adipose tissue. Prog Lipid Res 38:225-248.

Tamori Y, Masugi J, Nishino N, Kasuga M (2002) Role of peroxisome proliferator-activated receptor-gamma in maintenance of the characteristics of mature 3T3-L1 adipocytes. Diabetes 51:2045-2055.

Tirard J, Gout J, Lefrançois-Martinex AM, Martinex A, Begeot M, Naville D (2007) A novel inhibitory protein in adipose tissue, the aldo-keto reductase AKR1B7: its role in adipogenesis. Endocrinology 148:1996-2005. 